\title{
Optically Pumped Mid-Infrared Stimulated Emission of ZnSe:Cr Crystals
}

\author{
V.Yu. Ivanov ${ }^{a}$, M. Godlewski ${ }^{a, b}$, A. Szczerbakow ${ }^{a}$, \\ A. OMEL'ChuK ${ }^{c}$, A. DaVydov ${ }^{d}$, N. ZhaVoronkov ${ }^{d}$ \\ AND G. RACIUKAITIS ${ }^{e}$ \\ ${ }^{a}$ Institute of Physics, Polish Academy of Sciences \\ al. Lotników 32/46, 02-668 Warsaw, Poland \\ ${ }^{b}$ Dept. of Mathematics and Natural Sciences College of Science \\ Cardinal S. Wyszyński University, Warsaw, Poland \\ ${ }^{c}$ Institute of Semiconductor Physics NASU \\ Prospekt Nauki 45 Kiev, 252028, Ukraine \\ ${ }^{d}$ Research Institute of Material Science, Zelenograd, Moscow, Russia \\ ${ }^{e}$ Institute of Physics, Savanoriu Ave., 231Vilnius, 02300 Lithuania

\begin{abstract}
We demonstrate that ZnSe: $\mathrm{Cr}$ is an excellent solid state laser material for mid-infrared region with a broad amplification band from $2.2 \mu \mathrm{m}$ to $3 \mu \mathrm{m}$, and with a high quantum efficiency of a radiative recombination. $46 \%$ external total efficiency and $56 \mathrm{~mW}$ threshold power were achieved, when pumped with a CW YAlO:Ho laser at resonant excitation conditions. Such laser system can have widespread applications in medicine as laser scalpels with regulated cutting depth. We further demonstrate a stimulated emission under Cr $2+$ to $1+$ photo-ionization pumping. The latter opens chances for laser emission under carrier injection conditions.
\end{abstract}

PACS numbers: 42.55.Px, 42.55.Rz, 42.62.Be, 78.55.Et

\section{Introduction}

Laser surgery, introduced in early 1960 s, uses a precisely focused beam of light to remove or vaporize tissue and control bleeding in a wide variety of non-invasive and minimally invasive procedures [1]. The three types of lasers are most commonly used in medical treatment. These are carbon dioxide $\left(\mathrm{CO}_{2}\right)$ laser, used primarily as a surgical laser (laser scalpel), Nd:YAG laser, which is capable of 
penetrating tissue more deeply than other lasers, and argon laser, used primarily in eye surgery, but also in photodynamic therapy.

There are several advantages of laser surgery. Most importantly, laser surgery procedures usually less bleeding than conventional surgery and reduces the risk of infection. Sealing off blood vessels and nerves reduces bleeding and the length of the recovery period [1]. There are however some limitations of presently used lasers. They do not allow the control of cutting depth, which in some cases is critical. The latter limitation can be removed by introducing a tunable laser in a spectral region of an exponential change of water absorption (human body consists mostly of water). This explains the search for tunable laser sources in 2-4 micrometers spectral range, as explained in Fig. 1, showing an absorption spectrum of water.

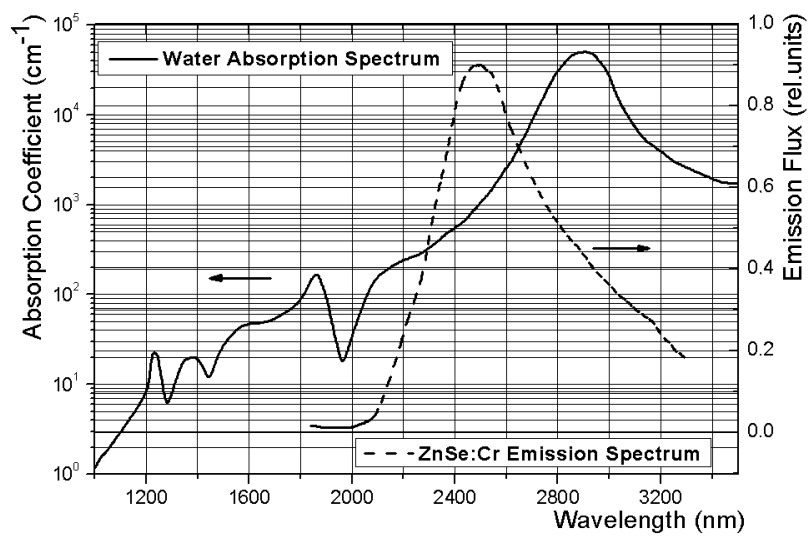

Fig. 1. Water absorption spectrum in mid-infrared spectral region together with $\mathrm{Cr}^{2+}$ intra-shell emission due to ${ }^{5} E$ to ${ }^{5} T_{2}$ emission observed in $\mathrm{ZnSe}: \mathrm{Cr}$.

Chromium doped II-VI semiconductors are attractive systems for such application. Two infrared emissions, with a maximum at about $0.95 \mu \mathrm{m}$ and $2.4 \mu \mathrm{m}$, are observed for $\mathrm{Cr}^{2+}$ intra-shell transitions. $2.4 \mu \mathrm{m}$ emission is relatively broad, which can result in optically pumped and tunable laser emission with wavelength of emission being suitable for application as a surgery laser, as indicated in Fig. 1. Figure 1 shows that $\mathrm{Cr}^{2+}{ }^{5} E$ to ${ }^{5} T_{2}$ intra-shell emission overlaps the spectral region of a strong water absorption, making possible a selection of an appropriate cutting depth, by selecting emission at a given water absorption rate.

Following this idea, a mid-infrared lasing action at about $2.5 \mu \mathrm{m}$ was achieved for several wide band gap II-VI semiconductors doped with chromium [2-7]. At present, ZnSe is the most promising host material for such mid-infrared laser. For ZnSe:Cr pulsed and also continuous-wave laser emissions under optical pumping were achieved with a power of emitted light above $1 \mathrm{~W}$. This opens a wide range of possible applications for this new mid-infrared laser system. 
In this work we demonstrate a very efficient $\mathrm{Cr}^{2+}$ stimulated emission upon optical pumping using either direct excitation or indirect one related to $2+$ to $1+$ chromium photo-ionization.

\section{Material related problems}

Despite several encouraging reports, Cr-related mid-infrared lasers were not commercialized yet. This was due to problems related to a growth of sufficiently good quality bulk ZnSe:Cr crystals. Bridgman-Stockbarger samples used to demonstrate laser emission contained too large densities of dislocations and of parasitic deep centers, and too large inhomogeneities of $\mathrm{Cr}$ concentration, including the presence of chromium precipitates. In the consequence, it was difficult to achieve repeatable laser parameters and a stable laser action.

In our approach we used high quality ZnSe crystals grown by Markov-Davydov physical transport vapor (PVT) method [8], which were doped with chromium during the growth. These were large size crystals $(50 \mathrm{~mm}$ diameter and $16 \mathrm{~mm}$ height) of a cylindrical shape with a chromium concentration up to a few times $10^{19} \mathrm{~cm}^{-3}$. An essential step was a post growth annealing of these crystals in zinc vapors at $940^{\circ} \mathrm{C}$. The annealing time was varied between 240 and 1000 hours. Such post-growth annealing helped to eliminate parasitic absorption bands related to deep centers (zinc vacancies) and improve optical properties of the samples. Sharp free excitonic spectra were observed in photoluminescence (PL) and reflectivity after the post-growth annealing, indicating a perfect quality of our samples. For stimulated emission experiments samples cut along cleavage faces were prepared.

\section{Stimulated emission upon direct $\mathrm{CW}$ optical pumping}

In our approach we used a simplified pumping system, as compared to that applied by DeLoach and co-workers [2, 3]. YAlO:Ho laser operating at $2013 \mathrm{~nm}$

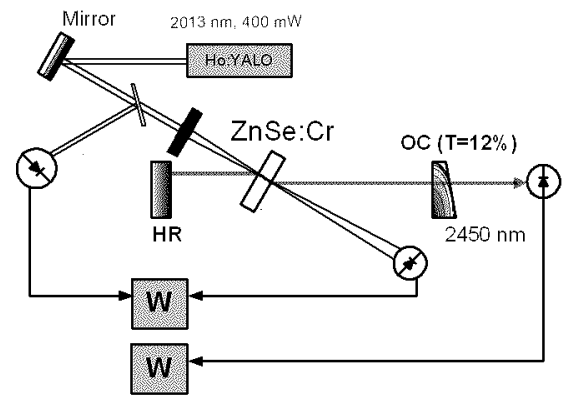

Fig. 2. Experimental set up used in the present study to pumped chromium mid-infrared emission under direct conditions. 


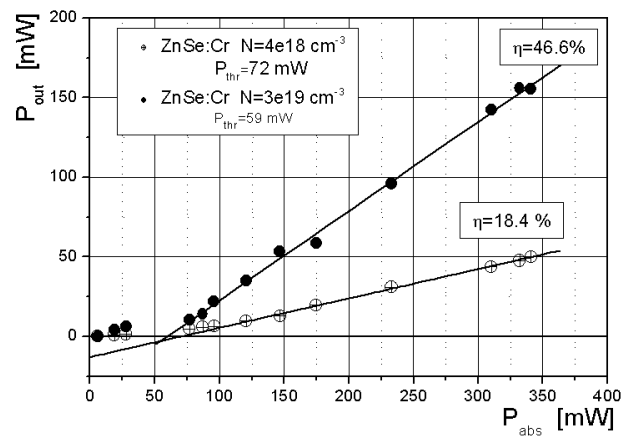

Fig. 3. Threshold dependence of a ${ }^{5} E$ to ${ }^{5} T_{2} \mathrm{Cr}^{2+}$ stimulated emission observed at room temperature for chromium doped $\left(5 \times 10^{19} \mathrm{~cm}^{-3}\right)$ ZnSe:Cr. Slope efficiency of a laser emission of $46.6 \%$ was achieved upon pumping normal to the laser cavity axis.

with a $0.4 \mathrm{~W}$ power was used for optical pumping with ZnSe:Cr crystal mounted in a Fabry-Perot laser cavity formed by a high reflector and $12 \%$ output coupler mirror optimized for $2450 \mathrm{~nm}$ wavelength (Fig. 2). We used a classical pumping configuration, i.e. emission was pumped normal to the laser cavity axis. A very bright stimulated emission was achieved upon optical pumping, with a high slope efficiency (Fig. 3) reaching 46\% for heavily Cr doped sample. Nearly $50 \%$ slope efficiency and $56 \mathrm{~mW}$ generation threshold power are very encouraging results, indicating that the system studied can find practical applications as a mid-infrared solid state laser system.

\section{Stimulated emission upon photo-ionization pumping}

Our previous PL experiments indicated that hole retrapping by a photo-excited $1+$ charge state of chromium proceeds via excited intra-shell states of chromium $\mathrm{Cr}^{2+}$ [9]. Thus, excitation of intra-shell emission of $\mathrm{Cr}^{2+}$ can be achieved upon processes changing the charge state of chromium ions. This observation suggested a possibility of efficient pumping of intra-shell emission by an indirect process related to $2+$ to $1+\mathrm{Cr}$ photo-ionization. $\mathrm{Cr}^{2+}$ is excited due to a subsequent hole retrapping from the valence band proceeding via one of $\mathrm{Cr}^{2+}$ intra-shell states.

There are two reasons why this process is of an interest. First, photo-ionization bands of transition metal ions are typically characterized by large oscillator strengths, i.e. the photo-excitation can be more efficient than the intra-shell excitation and, thus, can result in a much more efficient optical pumping of a mid-infrared emission. Second, if efficient, this pumping process opens the chance for laser emission upon carrier injection, but as well, the possibility of constructing compact laser systems, due to the availability of compact green/blue color laser diodes.

In Fig. 4 we show an experimental set up used by us to demonstrate laser emission upon photo-ionization pumping. A tunable optical parametric oscilla- 


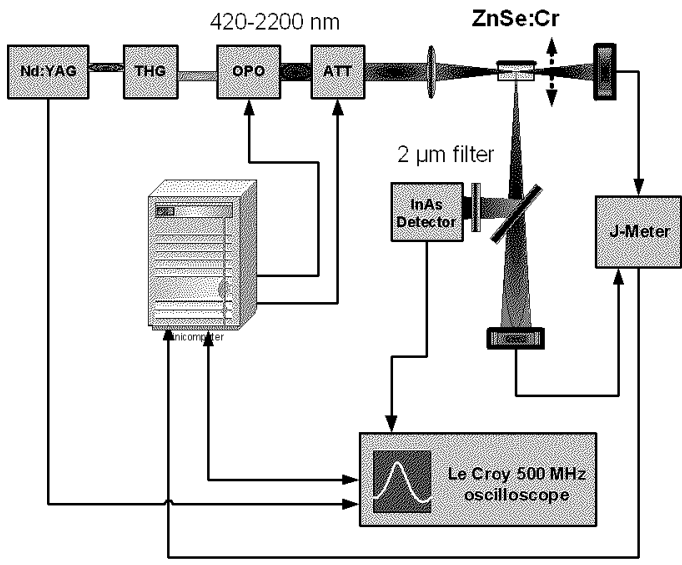

Fig. 4. Experimental set up used in the present study to pumped chromium mid-infrared emission under photo-ionization conditions.

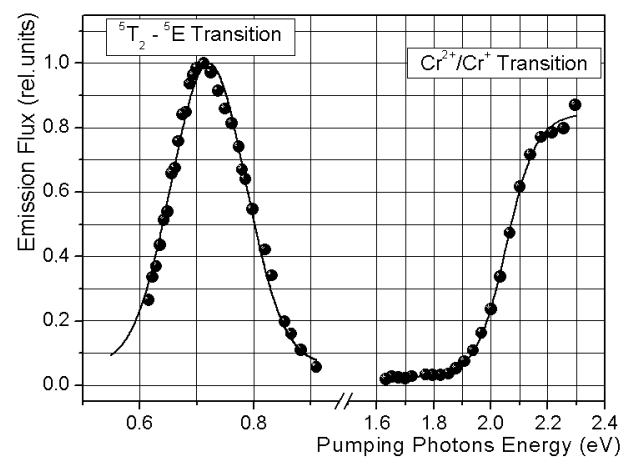

Fig. 5. Excitation spectra of ${ }^{5} \mathrm{E}$ to ${ }^{5} \mathrm{~T}_{2} \mathrm{Cr}^{2+}$ intra-shell emission in ZnSe:Cr measured at room temperature using tunable OPO system pumped with third harmonic YAG:Nd pulsed laser.

tor (OPO) system (420-2200 nm) pumped by a third harmonic of a pulsed YAG:Nd laser was used for optical pumping. A very bright emission was observed at such pumping conditions, despite the fact that we used a fairly primitive laser cavity. We evaporated a thin gold film as a high reflector mirror directly on a cleavage face of $\mathrm{ZnSe}$ crystal. As an output mirror acted the opposite face of the crystal with $46 \%$ transmission. We observed a threshold-like dependence on pumping density, with the threshold power only slightly larger than that observed upon a direct pumping. Slope efficiency was larger than that upon direct intra-shell pumping.

By tuning the pumping energy with OPO system (from $0.6 \mathrm{eV}$ to $2.4 \mathrm{eV}$ ) we could measure excitation bands of a mid-infrared emission. In addition to a direct (resonant) excitation $\left({ }^{5} T_{2}\right.$ to ${ }^{5} \mathrm{E}$, at $0.6 \mathrm{eV}$ to $1.3 \mathrm{eV}$ ) the second band starting 
at about $1.8-1.9 \mathrm{eV}$ was observed. This excitation band (shown in Fig. 5) follows chromium $2+$ to $1+$ photo-ionization, studied by us separately with photo-ESR [10]. This is the first evidence for optical pumping of a mid-infrared stimulated $\mathrm{Cr}^{2+}$ intra-shell emission upon a photo-ionization transition.

\section{Conclusions}

We demonstrate that $\mathrm{ZnSe:Cr}$ is a suitable solid state laser material for a tunable mid-infrared emission in a spectral range attractive for applications in medicine as a surgery laser. A stimulated emission upon photo-ionization pumping is demonstrated for the first time. The latter pumping mechanism of the emission opens two possibilities. First, a compact laser system can be constructed, using compact green or blue color laser diodes for ZnSe:Cr pumping. Second, the laser action upon carrier injection seems to be possible.

\section{References}

[1] Gale Encyclopedia of Medicine, Gale Research, 1999.

[2] L.D. DeLoach, R.H. Page, G.D. Wilke, S.P. Payne, W.F. Krupke. IEEE J. Quant. Electron. 32, 885 (1996).

[3] R.H. Page, K.I. Schaffers, L.D. DeLoach, G.D. Wilke, F.D. Patel, J.B. Tassano, Jr., S.A. Payne, W.F. Krupke, Kuo-Tong Chen, A. Burger, IEEE J. Quant. Electron. 33, 609 (1997).

[4] S. Bhaskar, P.S. Dobal, B.K. Rai, R.S. Katiyar, H.D. Bist, J.-O. Ndap, A. Burger, J. Appl. Phys. 85, 439 (1999).

[5] A. Burger, K. Chattopadhyay, J.-O. Ndap, X. Ma, S.H. Morgan, C.I. Rablau, C.-H. Su, S. Feth, R.H. Page, K.I. Schaffers, A. Payne, J. Cryst. Growth 225, 249 (2001).

[6] A.V. Podlipensky, V.G. Shcherbitsky, N.V. Kuleshov, V.I. Levchenko, V.N. Yakimovich, M. Mond, E. Heumann, G. Huber, H. Kretschmann, S. Kuck, Appl. Phys. $B$ 72, 253 (2001).

[7] E. Sorokin, I.T. Sorokina, Appl. Phys. Lett. 80, 3289 (2002).

[8] E.V. Markov, A.A. Davydov, Izv. Akad. Nauk SSSR, Neorg. Mater. 7, 575 (1971) (in Russian).

[9] V.Yu. Ivanov, T.P. Surkova, M. Godlewski, N. Zhavoronkov, A.R. Omelchuk, Phys. Status Solidi B 229, 355 (2002).

[10] V.Yu. Ivanov, Yu.G. Semenov, M. Surma, M. Godlewski, Phys. Rev. B 54, 4696 (1996). 